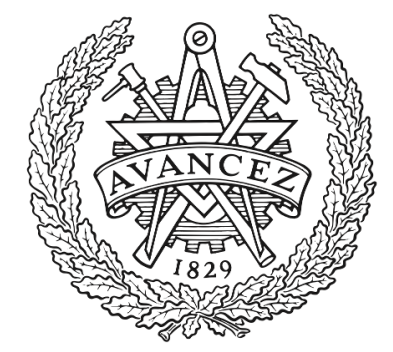

\title{
CHALMERS
}

UNIVERSITY OF TECHNOLOGY

\section{Performance of Probabilistic Shaping Coherent Channels in Hybrid Systems}

Downloaded from: https://research.chalmers.se, 2023-04-26 02:09 UTC

Citation for the original published paper (version of record):

Villafani Caballero, D., Mirani, A., Åhlfeldt, H. et al (2020). Performance of Probabilistic Shaping Coherent Channels in Hybrid Systems. International Conference on Transparent Optical Networks, 2020-July: 1-3. http://dx.doi.org/10.1109/ICTON51198.2020.9203055

N.B. When citing this work, cite the original published paper. 


\title{
Performance of Probabilistic Shaping Coherent Channels in Hybrid Systems
}

\author{
Diego Villafani**, Ali Mirani*, Henrik Åhlfeldt**, Jochen Schröder*, Magnus Karlsson* and \\ Peter Andrekson* \\ * Fiber Optic Communications Research Center (FORCE), Chalmers University of Technology, Gothenburg, \\ Sweden \\ ** Infinera, Stockholm, Sweden \\ E-mail: diego.villafani@infinera.com
}

\begin{abstract}
We present the performance comparison of probabilistically shaped 64QAM with uniform 16/32QAM in hybrid systems. The results show that the PS signal can increase the maximum reach and is more tolerant to strong nonlinear interference. Nevertheless, when the nonlinear interference is weak, uniform formats can provide higher performance.

Keywords: Coherent detection, probabilistic shaping, hybrid systems, wavelength division multiplexing.
\end{abstract}

\section{INTRODUCTION}

The upgrade of wavelength division multiplexing (WDM) optical metro networks from widely deployed 10 Gbps channels with nonreturn to zero (NRZ) on-off keying (OOK) modulation formats to $100 \mathrm{Gbps}$ coherent channels has been ongoing during the last years [1]. Moreover, the development of $200 \mathrm{Gbps}$ and $400 \mathrm{Gbps}$ transceivers, that uses higher order modulation formats and/or higher baud rate signals, has been investigated as the near future solution for the capacity upgrade of metro networks [2]. However, it is well known that when the coherent channels are inserted in legacy WDM links with dispersion compensation modules (DCM) in every span and co-propagating OOK channels, the transmission performance is degraded due to the increased contribution of nonlinearities. These systems, which include both the coherent and OOK channels, are typically called hybrid systems and have been extensively studied [3, 4].

One approach to make coherent channels more tolerant to nonlinear interference is to shape the signal constellation. This method is also used to approach the channel capacity and transmit more information per symbol. In order to do this, signal shape should mimic a certain distribution which maximizes the channel capacity. For additive white Gaussian noise (AWGN) channels, this distribution in known to have a Gaussian probability density function and there is $1.53 \mathrm{~dB}$ signal to noise ratio (SNR) gap between the channel capacity and what can be achieved by conventional uniform modulation formats [5]. Two main methods to shape the signal are probabilistic shaping (PS) and geometric shaping (GS). In both scenarios, power efficiency, reach increase and tolerance to system impairments have been reported [5,6]. One of the advantages of PS is its compatibility with the conventional quadrature amplitude modulation (QAM) systems and also easy rate control without affecting the structure of the forward error correction (FEC) and baud rate [7]. PS allows to have close approximation of Gaussian distribution and reduce the losses caused by shaping in conventional QAM formats [8]. In fiber optic communications, Maxwell-Boltzmann (MB) distribution is applied to improve the transmission reach. Furthermore, its effect on nonlinear interference and effective SNR have been studied and compared with other distributions [6,9]. Depending on the reference modulation format and transmission rates, gains between 7 to 40 percent have been shown [8].

A trade-off for higher order modulation formats is that they will require higher optical signal to noise ratio (OSNR) values for a specific bit error rate (BER) threshold, therefore reducing the maximum possible reach of the system. Furthermore, increasing the baudrate implies an increase in the costs of the electronics that is also not desired. The use of PS signals in 200Gbps coherent transceivers can reduce the OSNR requirements and increase the capacity and reach without the necessity of using higher bandwidth electronics and it has been successfully proved by J. Li et.al. in [10]. Nevertheless, the performance comparison of PS signals with uniform modulations in hybrid systems is still missing from reported studies. In this paper we evaluate the performance of a 240 and $300 \mathrm{Gbps}$ PS signal modulated with polarization multiplexing (PM) PS64QAM with 4 and 5 bits per symbol source entropy, and compare it with uniform PM-16/32QAM in hybrid systems. As a bench mark, the single channel transmission is simulated, and later, we increase the number of $10 \mathrm{Gbps}$ OOK channels in the system and analyse the performance, additionally, the maximum reach is investigated.

\section{System Simulation}

The PS constellation points are generated according to the MB distribution with the symbol probability distribution function of

$$
p_{X}\left(x_{i}\right)=e^{-v\left|x_{i}\right|^{2}} / \sum_{x \in X} e^{-v|x|^{2}},
$$




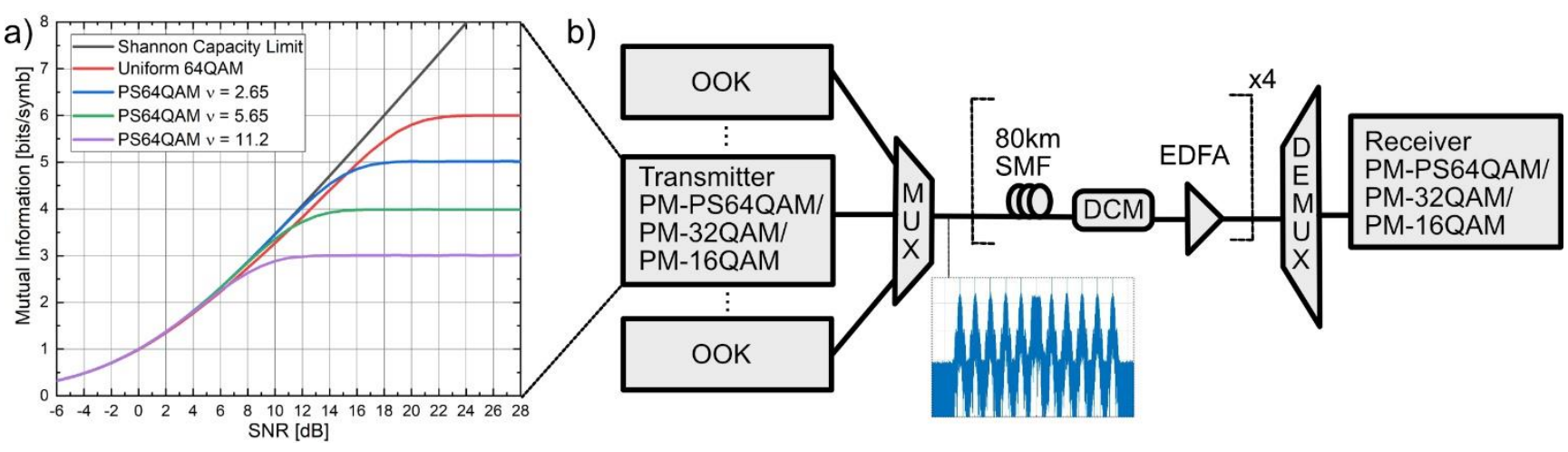

Figure 1: a) MI vs SNR for PS64QAM signal with different $n$ values. b) Simulation Setup.

where $x_{i}$ is the symbol coordinate in the complex plane, $\mathcal{X}$ is the source alphabet, |.| stands for amplitude of a complex number and $v$ is the shaping factor. Varying the shaping factor will result in different source entropy values and this help us to have a fair comparison between the PS signal and the uniform modulation format. We choose the shaping factor value such that the PS signal will have the source entropy of 4 and 5 bits per symbol that is equal to the source entropy for uniform 16QAM and 32QAM, respectively. Figure 1a shows the mutual information (MI) versus SNR in an AWGN channel where we variate the shaping factor of the MB distribution using a 64QAM modulation to obtain different source entropy values.

Figure $1 \mathrm{~b}$ shows the simulation setup for the performance evaluation of a PS signal in a hybrid system with $10 \mathrm{Gbps}$ NRZ OOK neighbours. The simulation consists of a transmitter that is able to modulate a uniform PM-16/32QAM signal and a PM-PS64QAM signal with different source entropy values in order to perform a fair comparison. The system simulation consists of a 30 Gbaud channel, Gray coded and shaped with a root-raised cosine (RRC) filter with 0.2 rolloff factor. The signal is modulated by an ideal IQ modulator and the laser phase noise is neglected. We multiplex the coherent channel in a $50 \mathrm{GHz}$ grid WDM system where the coherent channel is surrounded by a variable number of 10 Gbps NRZ OOK channels. Each data channel is generated by a decorrelated pseudo random bit sequence (PRBS) and a total of $2^{16}$ symbols are used with 64 times oversampling factor. The link consists of 4 spans of $80 \mathrm{~km}$ single mode fiber (SMF) with ideal DCM and loss compensation after each span. The attenuation in the SMF is $0.2 \mathrm{~dB} / \mathrm{km}$, the dispersion coefficient is $17 \mathrm{ps} / \mathrm{nm} / \mathrm{km}$ and the nonlinear coefficient is $1.371 / \mathrm{W} / \mathrm{km}$. The erbium doped fiber amplifier (EDFA) noise figure is $5 \mathrm{~dB}$. Fiber propagation is solved by using the split step Manakov model and neglecting polarization mode dispersion (PMD). At the receiver, we demultiplex the coherent channel with a $50 \mathrm{GHz}$ Gaussian optical filter and later use a RRC filter with the same characteristics as in the transmitter. A pilot aided digital signal processing (DSP) is used and includes the constant modulus algorithm (CMA) for polarization recovery and the pilot-based carrier phase estimation (CPE) with $3 \%$ overhead. We consider the OOK channels with 45 degrees state of polarization and a DCM that matches the accumulated dispersion at every span to have the worst case scenario with the highest nonlinear penalty [11].

\section{Simulation Results}
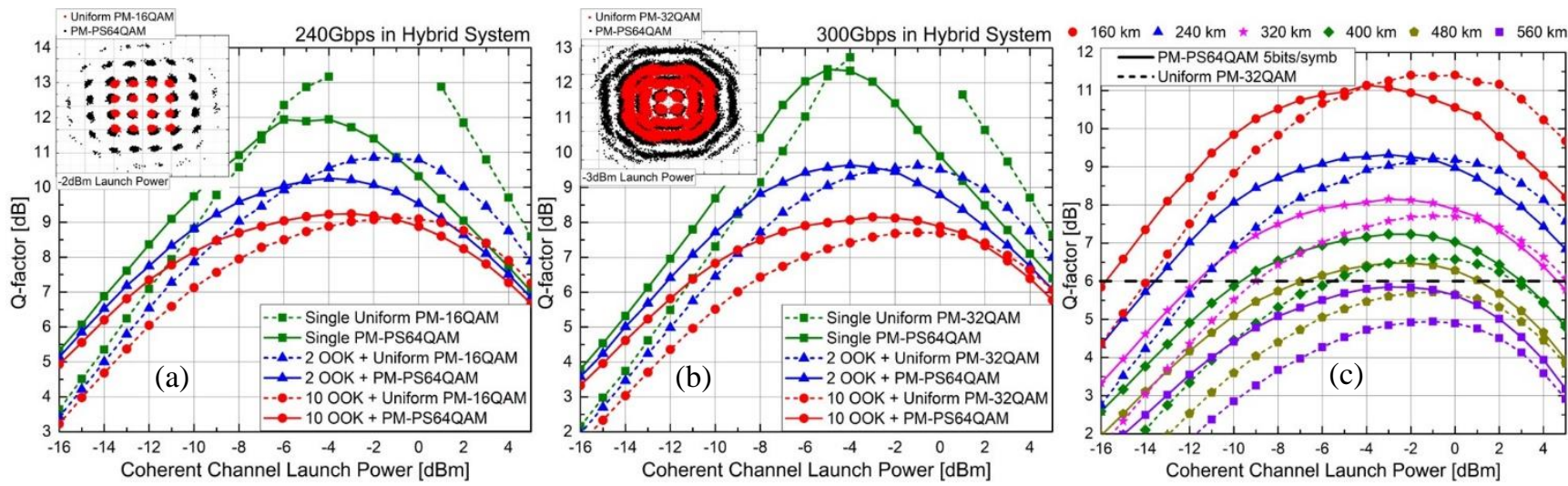

Figure 2: Performance comparison between uniform and PS signals in a hybrid system with different number of NRZ OOK neighbor channels. a) 240 Gbps Uniform PM-16QAM and PM-PS64QAM. b) 300 Gbps Uniform PM-32QAM and PM-PS64QAM. c) Maximum reach for 300 Gbps with 10 NRZ OOK neighbor channels

We started investigating the performance of a 240 Gbps uniform PM-16QAM versus a PM-PS64QAM in a hybrid system. Fig. 2a shows the performance evaluation in terms of the Q-factor versus the coherent channel launch power, where we first simulate the transmission of a single coherent channel. The results for a single channel show that the PM- 
PS64QAM has a better performance in the linear regime (up to -7 $\mathrm{dBm}$ ), but the uniform PM-16QAM signal is able to achieve a higher Q-factor in the nonlinear regime. This can be understood from the inset of Fig. 2a where the normalized constellation for $-2 \mathrm{dBm}$ launch power is shown. Here we observe that symbols with higher amplitude are used in the PS signal compared to the uniform signal, and this higher amplitude symbols are more affected by the nonlinear phase shift, resulting in a lower Q-factor. Later, we increase the number of OOK channels in the system to 2 and 10 (1 and 5 per side), where the launch power of the OOK channels is maintained at $-3 \mathrm{dBm}$, this power is suitable to achieve an error free transmission in the proposed system when $11 \mathrm{OOK}$ channels are considered. The results show that as we increase the number of OOK channels in the system, the nonlinear penalty reduces the maximum achievable Q-factor at each modulation format, but the performance in the uniform signal decreases faster than for the PS signal, showing the higher tolerance to strong nonlinear interference of the PS signal. Figure 2 a also shows that when 10 OOK channels are co-propagating, the PM-PS64QAM signal can achieve a slightly higher Q-factor than the uniform PM-16QAM signal by using $2 \mathrm{~dB}$ less optical launch power.

Figure 2b shows the performance of a 300 Gbps uniform PM-32QAM versus a PM-PS64QAM. The results show that as we increase the number of co-propagating OOK channels in the system to 2, the PS signal can achieve the same Q-factor as the uniform signal and requires $3 \mathrm{~dB}$ less optical launch power. The results also show, that as we increase the number of OOK channels to 10, the uniform signal is more degraded than the PS signal and a higher Q-factor is achieved by the PS signal. The inset of Fig. $2 b$ shows the constellation for the PM-PS64QAM and uniform PM-32QAM signals at $-3 \mathrm{dBm}$ launch power. It is clear from the received constellation that the lower amplitude symbols are less affected by the nonlinear interference. Furthermore, the probability of receiving a low amplitude symbol in the PS signal is much higher than the probability of receiving a low amplitude symbol in the uniform signal. This results in the PS signal achieving a higher Q-factor and a higher tolerance to strong nonlinear interference. Figure 2c shows the performance at different reach extensions for a 300 Gbps channel where we compare the PM-PS64QAM (solid line) with a uniform PM-32QAM (dashed line). The results show that the PS signal is able to achieve a higher Q-factor after $240 \mathrm{~km}$. As an example, if the FEC threshold is considered at $6 \mathrm{~dB}$ Q-factor, the PS signal can achieve $480 \mathrm{~km}$ reach with $0.5 \mathrm{~dB}$ Q-factor margin and the uniform signal is only able to achieve $400 \mathrm{~km}$ reach with $0.6 \mathrm{~dB}$ Q-factor margin, resulting in a $20 \%$ reach extension.

\section{Conclusions}

We have compared the performance of a PM-PS64QAM signal with a uniform PM-16/32QAM signal in a hybrid system. The results show that the PM-PS64QAM signal is more tolerant to strong nonlinear interference due to the high probability of sending low amplitude symbols and can provide a higher performance with lower launch power. Nevertheless, in cases where the nonlinear interference is weak, the uniform signal can achieve higher performance due to the higher amplitude symbols used in the PM-PS64QAM. We also show that when a FEC threshold of $6 \mathrm{~dB}$ Q-factor is considered, a $20 \%$ reach increase can be achieved by the PM-PS64QAM signal.

\section{ACKNOWLEDGEMENTS}

This work was supported by the Swedish Governmental Agency for Innovation Systems project EFFCOM.

\section{REFERENCES}

[1] H. Adams: Trends in Metro Optical Networks, March 2018.

[2] H. Zhang et al.: Real-time transmission of $16 \mathrm{~Tb} / \mathrm{s}$ over 1020km using 200Gb/s CFP2-DCO, Opt. Express, vol. 26, no. 6, pp. 6943-6948, 2018.

[3] D. Rafique et al.: Impact of nonlinear fibre impairments in $112 \mathrm{~Gb} / \mathrm{s}$ PM-QPSK transmission with $43 \mathrm{~Gb} / \mathrm{s}$ and $10.7 \mathrm{~Gb} / \mathrm{s}$ neighbours, ICTON, 2010.

[4] S. Searcy et al.: Experimental study of cross-phase modulation reduction in hybrid systems with copropagating 100G PM-QPSK and 10G OOK, Opt. Express, vol. 21, no. 25, pp. 31483-31491, 2013.

[5] Z. Qu et al.: On the probabilistic shaping and geometric shaping in optical communication systems, IEEE Access, vol. 7, pp. 21454-21464, 2019.

[6] J. Renner et al.: Experimental comparison of probabilistic shaping methods for unrepeated fiber transmission, JLT, vol. 35, no. 22, pp. 4871-4879, 2017.

[7] F. Buchali et al.: Experimental demonstration of capacity increase and rate-adaptation by probabilistically shaped 64-QAM, ECOC, 2015.

[8] D. Pilori et al:: Maximization of the achievable mutual information using probabilistically shaped squared-QAM constellations, OFC, 2017.

[9] A. Mirani et al.: Comparison of uniform cross QAM and probabilistically shaped QAM formats under the impact of transmitter impairments, Proc. European Conf. Optical Communication (ECOC), 2019.

[10] J. Li et al.: Field trial of probabilistic-shaping programmable real-time 200-Gb/s coherent transceivers in an intelligent core optical network, ACP, 2018.

[11] J. Renaudier et al.: Performance comparison of 40G and 100G coherent PDM-QPSK for upgrading dispersion managed legacy systems, OFC, 2009. 